\title{
Transcriptional responses to polycyclic aromatic hydrocarbon-induced stress in Arabidopsis thaliana reveal the involvement of hormone and defense signaling pathways
}

\author{
David Weisman ${ }^{\dagger 1}$, Merianne Alkio² and Adán Colón-Carmona*1
}

\begin{abstract}
Background: Polycyclic aromatic hydrocarbons (PAHs) are toxic, widely-distributed, environmentally persistent, and carcinogenic byproducts of carbon-based fuel combustion. Previously, plant studies have shown that PAHs induce oxidative stress, reduce growth, and cause leaf deformation as well as tissue necrosis. To understand the transcriptional changes that occur during these processes, we performed microarray experiments on Arabidopsis thaliana L. under phenanthrene treatment, and compared the results to published Arabidopsis microarray data representing a variety of stress and hormone treatments. In addition, to probe hormonal aspects of PAH stress, we assayed transgenic ethyleneinducible reporter plants as well as ethylene pathway mutants under phenanthrene treatment.

Results: Microarray results revealed numerous perturbations in signaling and metabolic pathways that regulate reactive oxygen species (ROS) and responses related to pathogen defense. A number of glutathione S-transferases that may tag xenobiotics for transport to the vacuole were upregulated. Comparative microarray analyses indicated that the phenanthrene response was closely related to other ROS conditions, including pathogen defense conditions. The ethylene-inducible transgenic reporters were activated by phenanthrene. Mutant experiments showed that PAH inhibits growth through an ethylene-independent pathway, as PAH-treated ethylene-insensitive etr 1-4 mutants exhibited a greater growth reduction than WT. Further, phenanthrene-treated, constitutive ethylene signaling mutants had longer roots than the untreated control plants, indicating that the PAH inhibits parts of the ethylene signaling pathway.

Conclusions: This study identified major physiological systems that participate in the PAH-induced stress response in Arabidopsis. At the transcriptional level, the results identify specific gene targets that will be valuable in finding lead compounds and engineering increased tolerance. Collectively, the results open a number of new avenues for researching and improving plant resilience and PAH phytoremediation.
\end{abstract}

\section{Background}

Polycyclic aromatic hydrocarbons (PAH) are a family of persistent, hydrophobic environmental toxins that originate from the incomplete combustion of carbon-based fuels as well as from the release of petroleum into the environment $[1,2]$. As PAHs are potent carcinogens in humans $[3,4]$, remediation of PAH contamination is an

\footnotetext{
* Correspondence: adan.colon-carmona@umb.edu

1 Department of Biology, University of Massachusetts Boston, 100 Morrissey

Blvd, Boston, MA 02125, USA

+ Contributed equally

Full list of author information is available at the end of the article
}

ongoing endeavor. Traditionally, removal of pollutants from soil is a disruptive and costly physical process; consequently, there is strong interest in applying phytoremediation, the use of plants to sequester, volatilize, or degrade pollutants $[5,6]$.

An idealized plant used for PAH removal would uptake large amounts of the pollutant into the root system, transport the molecules to cellular compartments, metabolize the pollutant, and utilize or volatilize the nontoxic byproducts. In practice, these processes are rate- or capacity-limited, thereby limiting the net removal of PAH 
from soil. Over time, stress from pollutants and their byproducts can cause cumulative plant damage, further reducing pollutant flux through the system. With the goals of identifying and relaxing these constraints, theoretical and applied research is ongoing. As an example of enhanced arsenic phytoremediation, a series of experiments identified limiting processes and introduced transgenic constructs into Arabidopsis, resulting in greatly increased uptake and tolerance of the pollutant [7-9]. Unlike in arsenic phytoremediation, where plant hyperaccumulation followed by harvesting is the goal, phytoremediation of PAHs could ultimately lead to complete degradation of the organic compounds.

Following PAH treatment, plants exhibit a variety of stresses. Previous studies have shown that PAHs cause trichome and leaf deformations, accumulation of $\mathrm{H}_{2} \mathrm{O}_{2}$, oxidative stress, cell death, upregulation of antioxidant systems, and reduced plant growth [1,10-14]. In many regards, these symptoms broadly resemble the pathogenic hypersensitive response (HR) [14]. While there is substantial evidence of oxidative stress, the signaling and biochemical changes leading to the complex PAH symptoms are unknown.

The phytohormone ethylene has long been known to play central roles in oxidative stress responses and cell death [15], in plant growth inhibition [16], and in abiotic as well as pathogen responses $[17,18]$. These broad parallels, as well as the observation that the ethylene-responsive gene GSTF2 is upregulated in PAH-treated Arabidopsis [14,19], suggest that ethylene signaling may play a role in the PAH stress response. To better understand these areas, this study performed DNA microarray experiments to measure global transcriptional changes in Arabidopsis when treated with the three-ringed $\mathrm{PAH}$ phenanthrene. In addition, possible roles of ethylene signaling were investigated using ethylene-responsive reporter plants, ethylene production mutants, ethylene signaling mutants, and exogenous application of an ethylene precursor.

\section{Results}

\section{Transcriptional responses to phenanthrene}

To assess differential transcript levels of PAH-treated Arabidopsis, microarray experiments were performed on wild type (WT) whole plants grown for 21 days on sterile medium containing $0 \mathrm{mM}$ or $0.25 \mathrm{mM}$ phenanthrene. The PAH treatment level is comparable to levels found in polluted land and water sites [10]. A statistically significant set of transcripts was selected using a Benjamini and Hochberg false discovery rate (FDR) of 0.05 . Of these, high-stringency biological relevance was defined as the genes with greater than two-fold change in either direction, resulting in 1031 phenanthrene-responsive transcripts that were analyzed further. The full microarray dataset is available in Additional File 1, and the differentially-expressed subset is available in Additional File 2.

To elucidate classes of transcripts affected by phenanthrene, gene ontology (GO) analyses were performed on the 1031 differentially-expressed genes. A summary of this analysis is available in Additional File 3. Complementing the GO analysis, MapMan figures (Additional File 4) were produced to visualize phenanthrene-induced changes in cellular processes. Additional File 5 highlights relevant transcriptional changes related to stress, hormone signaling, and other selected processes.

A striking feature is the downregulation of photosynthesis-related mRNA levels (Additional File 3, Additional File $4 \mathrm{a}, \mathrm{b})$. In concert with the reduced photosynthesis, chlorophyll and carotenoid biosynthesis as well as protein targeting to the chloroplasts were reduced (Additional File 3, Additional File 4c,d). Downregulated processes further included protein biosynthesis and gluconeogenesis (Additional File 3).

Of the differentially-expressed transcripts, there is a strong overrepresentation of genes involved in biotic and abiotic stresses, oxidative stress, wounding, immunity, and defense responses (Additional File 3, Additional File $4 \mathrm{e})$. For instance, the genes coding for the ethyleneinducible defense response proteins $P D F 1.2 a$ and PDF1.2b [20] were strongly upregulated on the microarray (Additional File 5$)$. The pathogenesis related (PR) gene $P R-1$, which is the marker gene for systemic acquired resistance (SAR) was upregulated over 200-fold. $P R-1$ is induced by salicylic acid (SA) but does not require ethylene or jasmonate [21]. Transcript levels of $P R-2,-3$ ( $B$-CHI, basic chitinase), -4 , and -5 were also increased by phenanthrene (Additional File 1, Additional File 5, and Additional File 6).

A variety of antioxidant and detoxification systems were affected (Additional File 3 and Additional File 4f). The transcript level of the arginine decarboxylase $A D C 2$, a key enzyme in polyamine synthesis, was increased on the PAH microarray (Additional File 2). Twelve microarray probes representing glutathione transferases (GST), enzymes that tag xenobiotics with glutathione for transport into the vacuole [22,23], reported significant increases (Additional File 2 and Additional File 5). For instance, the GST AtGSTU24 was upregulated on phenanthrene. Additionally, the microarray probe that recognized AtGSTF2 (At4g02520) and AtGSTF3 (At2g02930) indicated a 3.7-fold increase of the transcripts on phenanthrene. Similarly, the probe that binds the GSTs At1g02920 and At1g02930 indicated 12-fold upregulation of these genes. Among the phenanthrene responsive GSTs, AtGSTU24 has previously been shown to be sharply and rapidly induced by the herbicides acetochlor and metolachlor, as well as the explosives 2,4,6trinitrotoluene and hexahydro-1,3,5-trinitro-1,3,5-triaz- 
ine [24]. Along similar lines, UDP-glucoronosyl and UDP-glucosyl transferase UGT74F2 (At2g43820, Additional File 5) was strongly upregulated by phenanthrene. This gene was constitutively upregulated in antioxidant loss-of-function mutants [25], which is consistent with upregulation in response to reactive oxygen species (ROS). Activation of the secretory system is further indicated by upregulation of protein targeting through the ER and the Golgi apparatus (Additional File 4d). Inversely, mRNA levels of several antioxidant genes were diminished (Additional File 4e). Downregulated mRNAs include the catalases (Additional File 1) CAT1, CAT3, as well as $C A T 2$ which is consistent with previous RT-PCR data [10]. The ascorbate peroxidases APX4 (Additional File 5) and TAPX (Additional File 2) as well as the superoxide-dismutase FSD1 (Additional File 5) were also downregulated on phenanthrene.

Expression levels of many hormone-responsive genes were changed: Generally, jasmonic acid (JA), SA, or abscisic acid responsive genes were induced, whereas gibberellic acid, brassinolide or auxin responsive genes were repressed (Additional File 3, Additional File 4f, Additional File 5). Expression of many typical ethylene-inducible genes was induced, including defensins, $H E L$, GSTs and basic chitinase (Additional File 5). However, other typical ethylene-responsive genes, such as $H L S 1$, were unaffected. Two genes of the ethylene biosynthesis pathway were downregulated: ACS6, an aminocyclopropane1-carboxylic acid (ACC) synthase, and $A C O 2$, an 1-aminocyclopropane-1-carboxylic acid (ACC) oxidase. Of the 145 putative ethylene-regulated AP2/EREBP transcription factor genes [26], 126 are represented on the microarray (Additional File 1), and mRNA levels of ten of these were more than two-fold affected by phenanthrene. Interestingly, the ethylene response factor ERF1-1, which integrates ethylene and JA signals [27], was significantly upregulated in the PAH dataset (Additional File 1). An overview of the transcriptional changes in hormonal and other regulatory processes is given in Additional File 3 and Additional File 4f.

\section{Comparison between phenanthrene and other stress and hormone treatments}

The gene ontology and MapMan analyses (Additional File 3 and Additional File 4e) of the transcriptional profile indicate that the PAH response shares commonality with biotic stress responses. Illustrating this relationship, Figure 1 compares the phenanthrene dataset to the treatment with the pathogenic fungus Botrytis cinerea, and indicates a strong correlation $(\rho=0.72)$ between the two treatments. In Figure 1, Quadrants I and III contain the transcripts that were jointly up- or downregulated on both treatments. The vast majority of the phenanthrene responsive transcripts fall into these categories. For

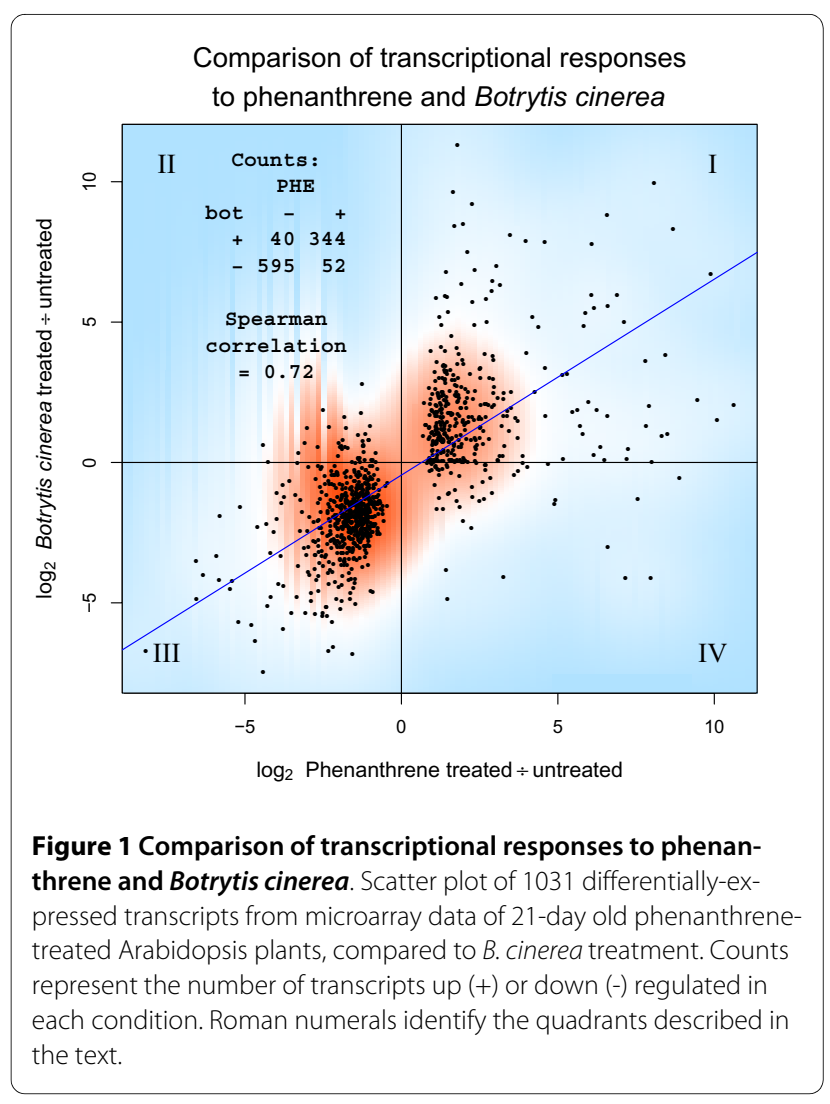

instance, the cell wall expansins AtEXP1, AtEXP8 [14], and $A t E X P 11$ were downregulated on both treatments (Quadrant III). Quadrant II contains transcripts that were downregulated by phenanthrene but upregulated by the $B$. cinerea fungal attack, and includes the ethylene biosynthesis gene ACS6. Inversely, Quadrant IV contains transcripts that are highly expressed on phenanthrene and diminished by the pathogen, including the cell wall expansin AtEXP4, AtNAP2 (POP1), which encodes a NAP-type ABC transporter, and At1g47400 of unknown function.

To further compare the PAH response with other experimental conditions, the phenanthrene dataset was clustered with a variety of published microarray datasets measuring responses to biotic, abiotic, chemical, and physical stresses as well as hormone and hormone inhibitor treatments. Table 1 shows correlations between the phenanthrene microarray and other experimental conditions. The heatmap in Figure 2 shows the results from clustering genes and experimental conditions. The complete dataset of the heatmap is available in Additional File 6 . The manifest clusters in the heatmap show strong similarity with various strains of Pseudomonas syringae, as well as the fungi B. cinerea and Erysiphe orontii. Ozone, osmotic, and oxidative stresses, as well as senescence, also correlated with the phenanthrene response. 
Table 1: Transcriptional correlations between phenanthrene and other treatments.

\begin{tabular}{|c|c|c|c|}
\hline Code & Treatment & Correlation & NASC \\
\hline PHE & Phenanthrene ( 3 w, 3 w) & 1.00 & \\
\hline bot & Botrytis cinerea (4 w, $48 \mathrm{~h})$ & 0.72 & 167 \\
\hline pst & P. syringae patovar tomato (5 w, $24 \mathrm{~h})$ & 0.71 & 330 \\
\hline o3 & Ozone (2 w, 6 h) & 0.67 & 26 \\
\hline avr & P. syringae avrRpm 1 (5 w, $24 \mathrm{~h})$ & 0.66 & 120 \\
\hline pha & P. syringae phaseolicola (5 w, 24 h) & 0.64 & 120 \\
\hline oss & Osmotic stress ( 2 w, 24 h; shoot) & 0.64 & 139 \\
\hline ps1 & P. syringae DC3000 (5 w, $24 \mathrm{~h})$ & 0.63 & 120 \\
\hline sen & None; senescence (mid flowering; leaves) & 0.62 & 52 \\
\hline pvi & P. syringae ES4326 (4 w, 48 h) & 0.60 & 168 \\
\hline sa & Salicylic acid (1 w, 3 h) & 0.55 & 192 \\
\hline eoi & Erysiphe orontii $(5 \mathrm{w}, 5 \mathrm{~d})$ & 0.52 & 169 \\
\hline oxs & Oxidative stress ( $2 \mathrm{w}, 24 \mathrm{~h}$; shoot) & 0.51 & 139 \\
\hline ag3 & $\mathrm{AgNO}_{3}(1 \mathrm{w}, 3 \mathrm{~h})$ & 0.48 & 188 \\
\hline gts & Genotoxicity ( 2 w, 24 h; shoot) & 0.46 & 142 \\
\hline uvs & UV radiation ( $2 \mathrm{w}, 24 \mathrm{~h}$; shoot) & 0.42 & 144 \\
\hline tib & 2,3,5-triiodobenzoic acid (TIBA; $1 \mathrm{w}, 3$ h) & 0.36 & 186 \\
\hline pav & P. syringae ES4326 avrRpt2 (4 w, 48 h) & 0.32 & 168 \\
\hline iaa & Indoleacetic acid (IAA; $1 \mathrm{w}, 3 \mathrm{~h})$ & 0.27 & 175 \\
\hline mja & Methyl jasmonate $(1 \mathrm{w}, 3 \mathrm{~h})$ & 0.26 & 174 \\
\hline ga3 & Gibberellic aid (1 w, 3 h) & 0.20 & 177 \\
\hline aba & Abscisic acid (1 w, $3 \mathrm{~h})$ & 0.19 & 176 \\
\hline $\mathrm{ctk}$ & Cytokinin (3 w, $3 \mathrm{~h})$ & 0.18 & 181 \\
\hline acc & 1-aminocycloprop. 1-carbox. acid (1 w, 3 h) & 0.18 & 172 \\
\hline pac & Paclobutrazol (1 w, $12 \mathrm{~h})$ & -0.01 & 185 \\
\hline avg & Aminoethoxyvinylglycine (1 w, 3 h) & -0.01 & 188 \\
\hline bra & Brassinolide $(1 \mathrm{w}, 3 \mathrm{~h})$ & -0.03 & 178 \\
\hline css & Caesium-137 (shoot; 3 w, 2 w) & -0.07 & 324 \\
\hline
\end{tabular}

In contrast with the phenanthrene-induced downregulation of ACS6, the transcript was upregulated by B. cinerea attack and in other biotic stresses, oxidative stress, O3, SA, genotoxicity, indoleacetic acid (IAA), TIBA (inhibitor of polar auxin transport) and AgNO3 (inhibitor of ethylene signaling) treatments. WRKY40, a member of a transcription factor family that frequently plays critical roles in stress responses [28], followed a similar pattern. bHLH101, a basic helix-loop-helix transcription factor, was sharply upregulated on the phenanthrene, O3, and genotoxicity microarrays, but little affected by the bacterial infections. AtOPT3, an oligopeptide transporter was similarly regulated.

Among the hormone treatment microarrays, the SA dataset had the strongest correlation with the phenanthrene data (Spearman correlation $\rho=0.55$, Table 1). In addition to $P R-1$ and other pathogen resistance (PR) genes, the phenanthrene microarray identified additional transcripts that indicate SA involvement. First, ICS1, an isochorismate synthase involved in SA biosynthesis, is normally induced by pathogen infection [29] and was upregulated on phenanthrene (Additional File 1). Second, the transcript of EDS5 (SID1), a MATE transporter nec- 


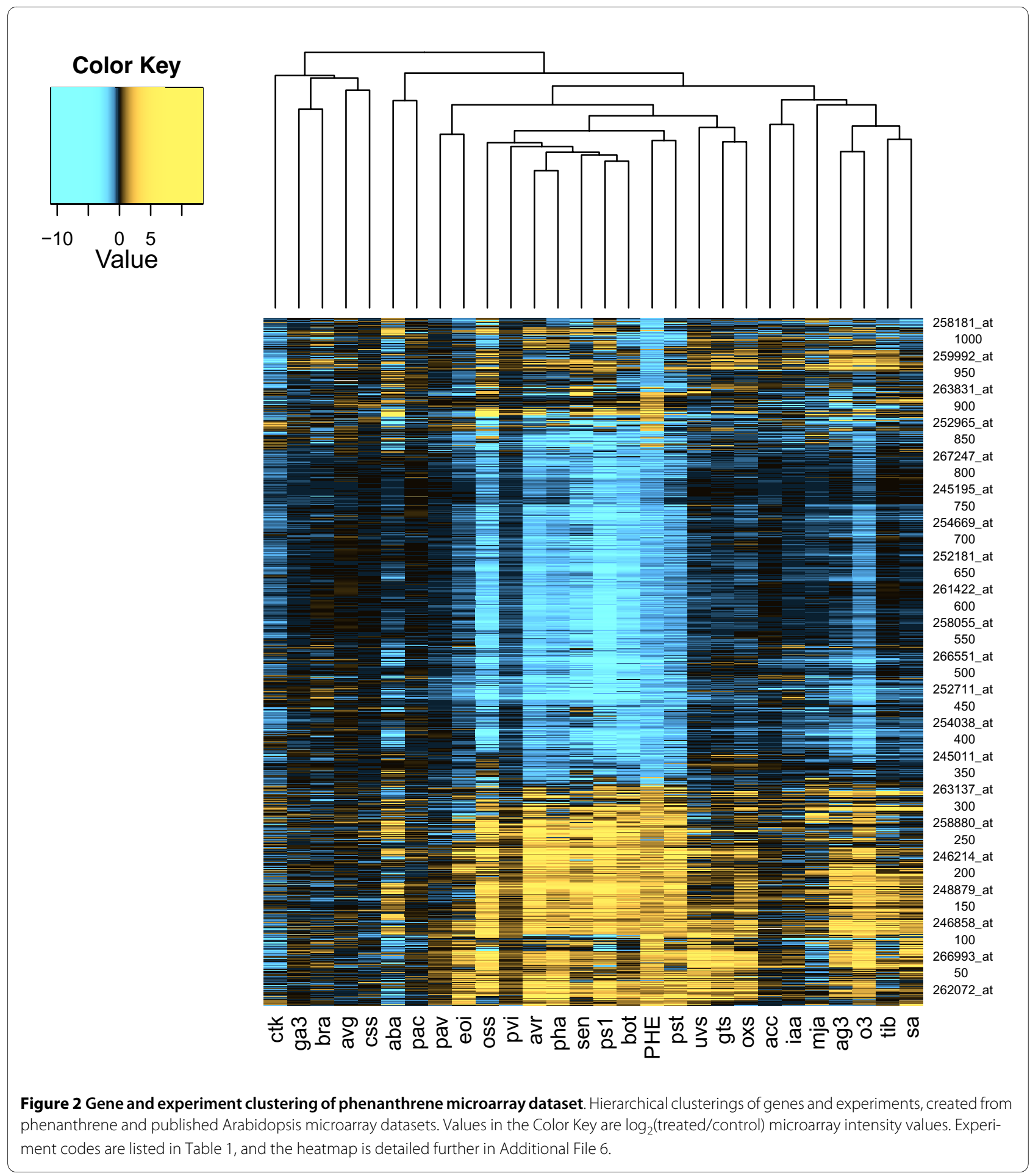

essary for SA signaling, was also upregulated by the $\mathrm{PAH}$ (Additional File 1), and is also induced in the $\mathrm{O}_{3}$, ultraviolet, and some biotic stress datasets. Finally, several SA early-response transcripts were induced on the phenanthrene and SA microarrays, including the UDP-glycosyl transferase $U G T 1$ and GST25.
Low correlations with the phenanthrene treatment were found for treatments with abscisic acid, the auxin transport inhibitor triiodobenzoic acid (TIBA), brassinolide, cytokinin, the auxin indoleacetic acid, the gibberellic acid biosynthesis inhibitor paclobutrazol (PAC), the ethylene precursor ACC, and the inhibitor of ethylene biosynthesis, aminoethoxyvinylglycine (AVG) (Table 1). 
However, treatment with $\mathrm{AgNO}_{3}$, which inhibits ethylene signaling [30], correlated noticeably with phenanthrene $(\rho=0.48)$. The inconsistency of the two ethylene inhibitors could be due to non-ethylene side-effects of $\mathrm{AgNO}_{3}$ or AVG. Analyzing the full set of $\sim 23 \times 10^{3}$ probes on the microarray, these two treatments produced a paradoxically low Spearman correlation coefficient of $\rho=0.21$, thereby supporting a side-effect hypothesis. Furthermore, the correlation between the $\mathrm{AgNO}_{3}$ and $\mathrm{O}_{3}$ microarray datasets was $\rho=0.60$, hinting that silver nitrate induced oxidative stress. Taken together, these data indicate that the similarities between phenanthrene and $\mathrm{AgNO}_{3}$ induced stress responses are not related to perturbed ethylene signaling.

\section{Analyses of transgenic ethylene-responsive GUS-reporter plants}

Ethylene is commonly known as a stress hormone. The microarray results clearly indicated involvement of ethylene-regulated genes in the phenanthrene response, but the downregulated ethylene biosynthesis transcripts $A C O 2$ and ACS6 suggested that the PAH reduced ethylene production. At the same time, comparisons of the phenanthrene data with ethylene inhibition and precursor spike-in datasets (AVG, $\mathrm{AgNO}_{3}$, and ACC in Table 1 and Figure 2) suggested that ethylene involvement was more nuanced than a global up- or down-regulation of ethylene signaling. To better understand this relationship, we analysed the role of ethylene under phenanthrene treatment more closely.

First, to observe localized effects of phenanthrene on ethylene signaling targets, we used the transgenic reporter plants CH5B::GUS and AtGSTF2::GUS, which indicate GUS expression driven by ethylene-inducible promoters from the bean basic chitinase [31] and AtGSTF2 genes [19], respectively. Activation of transcription from the $C H 5 B$ promoter in Arabidopsis leaves requires ethylene signaling through the ethylene receptor ETR1 [31]. In contrast, while being responsive to ethylene, the AtGSTF promoter can also be activated through an ETR1-independent mechanism after treatment with glutathione, paraquat, copper, and naphthalene acetic acid (NAA) [19]. Figure 3 shows reporter gene expression in both lines when grown in long days in the presence of phenanthrene. In both lines, the reporter expression occurred in scattered patches on the leaf blades. These spatial patterns are similar to the patterns of necrotic lesions induced by phenanthrene [14]. To dissect the contributions of phenanthrene and ethylene in activating these promoters, the two reporter lines were grown in the dark for $4 \mathrm{~d}$ while treated with combinations of phenanthrene and ACC. Figure 4 shows that in both lines, compared to the untreated controls (Figure 4A and 4E), PAH treatment upregulated GUS expression (Figure $4 \mathrm{C}$ and

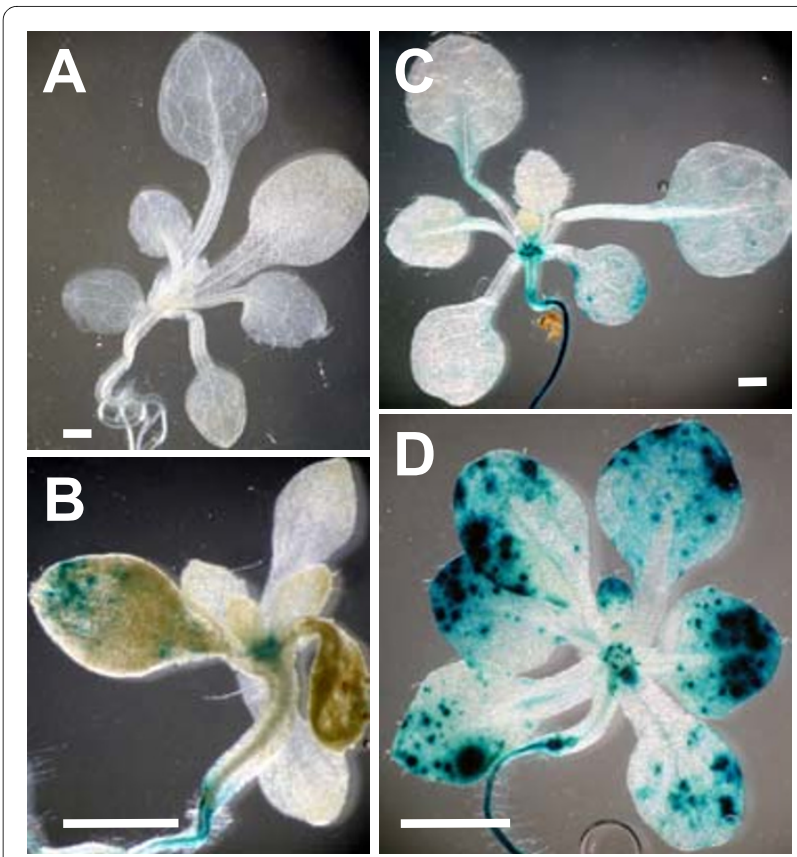

Figure 3 Ethylene reporter gene expression in plants treated with phenanthrene and grown in long day light. Histochemical staining of GUS activity in CH5B:: GUS (A, B), AtGSTF2::GUS (C, D) transgenic Arabidopsis plants in absence $(A, C)$ or presence $(B, D)$ of phenanthrene. Plants were grown in long days for $14 \mathrm{~d}$. Seedlings were stained for $15 \mathrm{~h}$ for GUS activity in staining buffer containing $2 \mathrm{mM} \mathrm{5-}$ bromo-4-chloro-3-indolyl-b-D-glucuronide. Scale bars $1 \mathrm{~mm}$.

4G). The treatments with ACC alone (Figure 4B and 4F) or in combination with phenanthrene (Figure 4D and $4 \mathrm{H}$ ) produced similar GUS expression patterns.

Although the histological GUS-assay is not quantitative, the relative intensity of staining can provide mean-

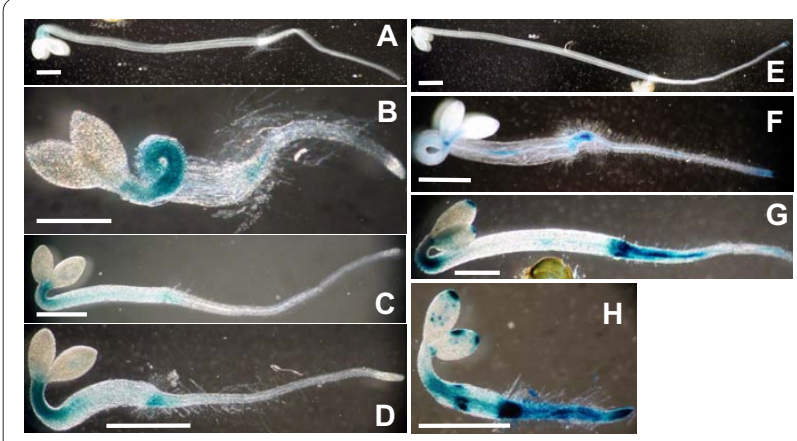

Figure 4 Ethylene reporter gene expression in plants treated with phenanthrene and ACC, and grown in the dark. Histochemical staining of GUS activity in CH5B::GUS (A-D) and AtGSTF2::GUS (E-H) transgenic Arabidopsis plants grown for $4 \mathrm{~d}$ in the dark. $\mathrm{A}$ and $\mathrm{E}, 0 \mathrm{mM}$ phenanthrene, $0 \mu \mathrm{M}$ ACC; $B$ and F, 0 mM phenanthrene, $20 \mu \mathrm{M} \mathrm{ACC;C}$ and $\mathrm{G}, 0.25 \mathrm{mM}$ phenanthrene and $0 \mu \mathrm{M} \mathrm{ACC}$; $\mathrm{D}$ and $\mathrm{H}, 0.25 \mathrm{mM}$ phenanthrene, $20 \mu \mathrm{M}$ ACC. Seedlings were stained for $15 \mathrm{~h}$ for GUS activity in staining buffer containing $2 \mathrm{mM}$ 5-bromo-4-chloro-3-indolylb-D-glucuronide. Scale bars $1 \mathrm{~mm}$. 
ingful information. When treated with phenanthrene, GUS expression was generally stronger in AtGSTF2::GUS plants than in the $C H 5 B:: G U S$ plants (Figure 3 and Figure 4). The strongest GUS activity in CH5B::GUS was due to ACC treatment (Figure 4B). In contrast, in AtGSTF2::GUS GUS activity was strongest in plants exposed to both ACC and phenanthrene (Figure $4 \mathrm{H}$ ).

Responses of ethylene mutants to phenanthrene treatment To further determine whether PAH stress involves ethylene signaling, we compared the phenotypes of several ethylene mutants to WT Arabidopsis grown on phenanthrene-containing media. The classic triple response of dark-grown seedlings grown in the presence of ethylene produces an exaggerated apical hook, a thickened, short hypocotyl, and a short root. Utilizing this behavior, ethylene signaling mutants and WT were grown in the dark to examine how the mutations affected phenanthreneinduced growth responses in seedlings. The mutants included the ethylene overproducer eto3 [32]; the ethylene-insensitive gain-of-function receptor mutant etr1-4 [33]; the mutant etr1-7 [34,35] which exhibits slightly enhanced ethylene sensitivity, and the etr1-6;etr2-3;ein44 [34] triple ethylene receptor mutant which exhibits constitutive ethylene signaling. Because the WT and ethylene signaling mutants differ in root and shoot lengths even under control conditions, absolute length comparisons under phenanthrene treatment are not meaningful between genotypes. To facilitate comparison, the relative length change within each genotype was defined as the ratio (\%) of phenanthrene-treated length to non-treated length. These response ratios were then compared between the genotypes.

Figure 5 shows phenanthrene-induced hypocotyl growth responses in dark-grown seedlings. Phenanthrene treatment reduced hypocotyl elongation in all plants except in eto3, in which hypocotyl length was unaffected. As a baseline, the hypocotyls of WT were $12.0 \pm 0.2 \mathrm{~mm}$ long on control medium, and $7.9 \pm 0.1 \mathrm{~mm}$ long on 0.5 $\mathrm{mM}$ phenanthrene, giving a response ratio of $66 \pm 1.3 \%$. In the ethylene-overproduction mutant eto3, the lengthreducing effect of phenanthrene was mitigated, producing hypocotyls as long as in the untreated control. Conversely, etr 1-4, the ethylene-insensitive mutant grew to only $40 \pm 4.8 \%$ of the length of the untreated mutant. The genotypes etr1-7 (response $60 \pm 7.6 \%$ ) and etr1-6;etr23; ein4-4 (response $67 \pm 2.2 \%$ ) did not differ significantly from WT in their hypocotyl responses to phenanthrene.

Root growth of phenanthrene-treated mutants differed markedly from the hypocotyl responses (Figure 6). Contrasting with the hypocotyl, root elongation of darkgrown WT was only marginally affected by phenanthrene, and the etr1-7 mutant was unaffected. Surprisingly, eto3 (response $174 \pm 12 \%$ ) and the triple mutant

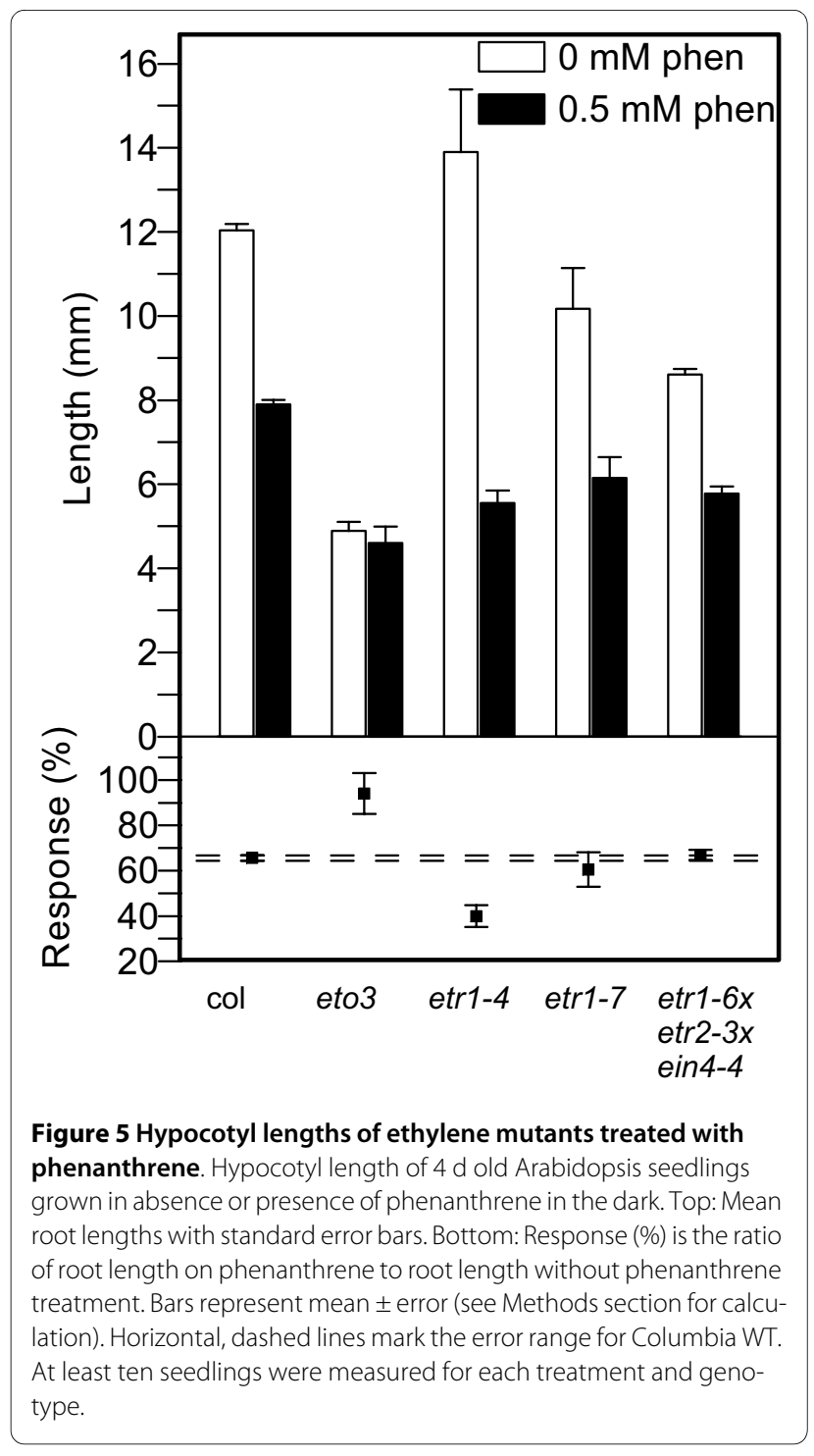

etr1-6; etr2-3;ein4-4 (response $187 \pm 7.4 \%$ ) grew even longer roots on phenanthrene than on control medium. In contrast, etr1-4 roots were significantly shorter on phenanthrene than on control medium (response $44 \pm$ $4.7 \%)$.

\section{Discussion}

Broadly, the response of Arabidopsis to phenanthrene is a complex perturbation of multiple systems, with a dominant theme of oxidative stress and similarities to pathogenic responses.

\section{Phenanthrene induces oxidative stress and a metabolic shift from anabolism to catabolism}

Consistent with physiological studies that associated PAH treatment with oxidative stress [10-12,14], transcripts related to oxidative stress were overrepresented among the phenanthrene responsive genes (Additional 


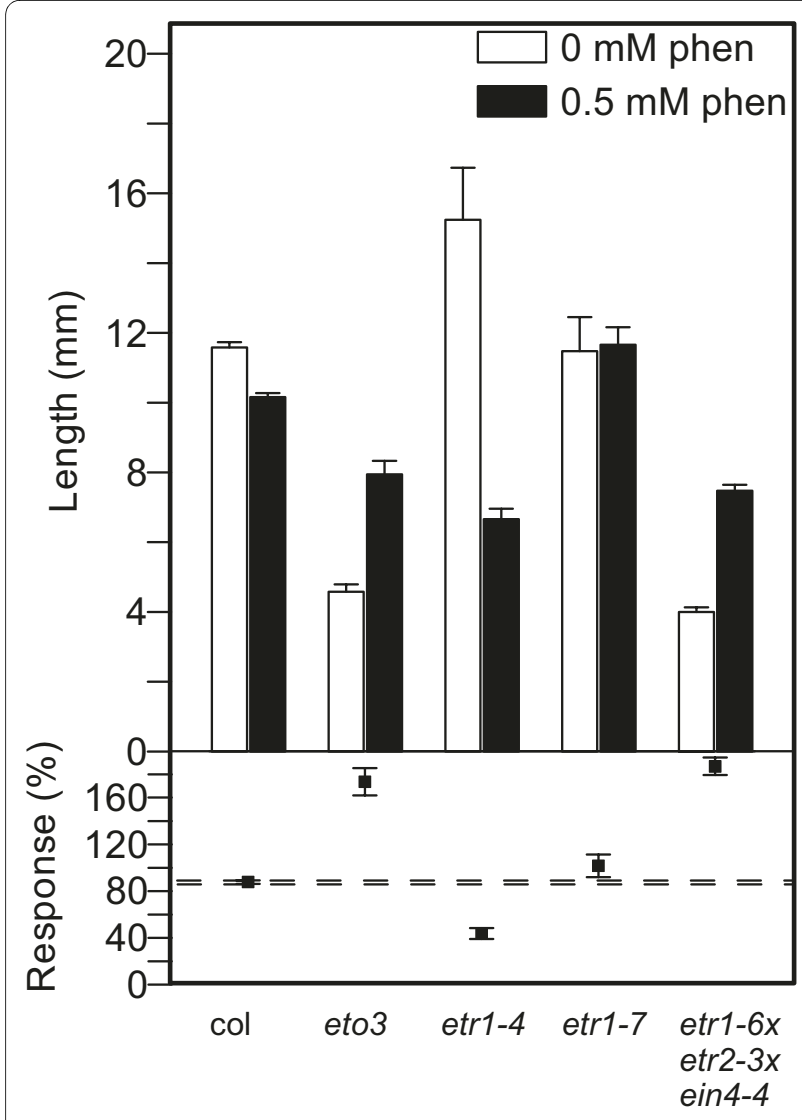

Figure 6 Root lengths of ethylene mutants treated with phenanthrene. Root length of 4-day old Arabidopsis seedlings grown in absence or presence of phenanthrene in the dark. For further explanations, see Figure 5 legend.

File 3 and Additional File 4e). In addition, polyamine levels and ADC enzyme activity were reported to increase in the aquatic plant Riccia fluitans when treated with phenanthrene [12], which is consistent with the present data that indicate an upregulation of $A D C 2$ mRNA.

At the systemic level, the microarray results bear strong resemblance to the transcriptional responses induced by fungal, bacterial pathogen, ozone or osmotic shock treatments (Figure 1, Figure 2, Table 1, and Additional File 4e). As the phenanthrene-treated plants were grown in sterile conditions, it is unlikely that the similarities to pathogen treatments were caused by confounding microbial effects. More likely, the unifying theme of these treatments is the production of ROS [15,36-40]. Following the initial oxidative burst, PAH-treated plants activate mechanisms similar to a pathogen defense including HR-like cell death [14] and induction of a battery of defense genes.

Similar responses have been described in ozone-treated plants, which also generate ROS and erroneously activate pathogen defense programs [15]. However, while oxidative stress was occurring under phenanthrene treatment, several antioxidant genes were downregulated. This sce- nario can occur when plants invoke a positive feedback loop that amplifies ROS to serve as signaling molecules $[28,41]$. An early perturbation of the redox network is clear as downregulation of catalase mRNA [10], as well as increased $\mathrm{H}_{2} \mathrm{O}_{2}$ levels and cell death [14], were detected within $12 \mathrm{~h}$ of PAH treatment. Along similar lines, prior work in Arabidopsis found CAT2 downregulated within three hours of $\mathrm{O}_{3}$ treatment [42]. Supporting the notion of ROS positive feedback activation, the respiratory burst oxidase AtRbohD was upregulated in phenanthrenetreated plants (Additional File 5), and was similarly upregulated by $\mathrm{O}_{3}$ and pathogenic attack conditions. Similarly, the tobacco ortholog NtrbohD was induced during an oxidative burst under $\mathrm{O}_{3}$ treatment [15]. The widespread destruction of chloroplast and mitochondrial membranes [10] may have injected additional ROS into the system.

PAH treatment caused downregulation of genes involved in photosynthesis and protein biosynthesis (Additional File 3 and Additional File 4a), which agrees with previous studies reporting overall diminished plant size and reduced chlorophyll levels [10,14]. Up-regulation of glycolysis and the citric acid cycle (Additional File 3 and Additional File 4a), as well as the similarity to the senescence microarray data (Table 1, Figure 2), further reveal a major metabolic shift from anabolism to catabolism. In addition, growth inhibition and the breakdown of the photosynthetic machinery are commonly observed ethylene effects [16].

\section{Phenanthrene interferes with hormone signaling networks} Results presented here suggest that the complex physiological PAH stress symptoms likely involve multiple hormone pathways, including SA, ethylene, JA, and abscisic acid (ABA). Furthermore, the GUS expression patterns in phenanthrene-treated CH5B::GUS and AtGSTF2::GUS lines suggest that ethylene and SA levels are locally elevated in PAH-stressed plant tissues (Figure 3 and Figure 4). The spatial patterns in leaves resemble previous observations of phenanthrene-induced, localized cell death and $\mathrm{H}_{2} \mathrm{O}_{2}$ accumulation [14], supporting the hypothesis that, in addition to SA, ethylene is involved in the development of the PAH symptoms. Interestingly, reporter activity was consistently more pronounced in PAH-treated AtGSTF2::GUS than in the CH5B::GUS transgenic plants (Figure 3 and Figure 4). This difference in GUS expression may be caused by a differential ethylene sensitivity of the two promoters. This explanation is plausible as the $C H 5 B$ promoter in transgenic Arabidopsis leaves is approximately an order of magnitude less sensitive to ethylene than the endogenous basic chitinase promoter [31]. A further explanation for the differential reporter levels is that the two transcriptional programs involve other sig- 
nals in addition to ethylene [19,31]. Indeed, SA signaling is necessary for strong AtGSTF2 induction by ethylene [43].

Analyses of quantitative growth responses of darkgrown ethylene mutants exposed to phenanthrene revealed further interesting interactions between phenanthrene and ethylene signaling. Without PAH, the ethylene overproducer eto3 and the constitutively-signaling triple mutant grew short hypocotyls and roots, consistent with the standard model of ethylene-induced growth reduction. However, when treated with phenanthrene, these two lines grew longer roots than on control medium, suggesting that the treatment inhibits ethylene signal transduction. This hypothesis is supported by the observations that the exaggerated apical hook, which is typical in ACC-treated dark-grown plants (Figure 4B and $4 \mathrm{~F}$ ), was absent in PAH-treated plants (Figure 4D and $4 \mathrm{H})$. This phenotype was frequently observed in WT plants treated with both ACC and phenanthrene (not shown). The observation that typical triple-response symptoms were attenuated under phenanthrene treatment suggests that the PAH negatively interferes with the ethylene signal transduction pathway or with ethylene biosynthesis in conditions of elevated ethylene levels or signaling. It has been proposed that ethylene can exhibit inhibiting or stimulating effect on growth, depending on the ethylene concentration [16]. Furthermore, the ethylene-insensitive etr1-4 mutant responded to the PAH with significantly stronger growth inhibition than the WT. This result clearly shows that the phenanthrene-induced growth reduction does not require ethylene signaling through the ETR1 receptor. Taken together, the mutant experiments suggest that ethylene is not required for the development of some of the PAH stress symptoms, and, phenanthrene inhibits some ethylene responses under conditions of elevated ethylene levels.

\section{Integrated model of PAH response in Arabidopsis}

With these and previous results taken in total, we propose a model of the PAH response in plants. Shortly following uptake, the PAH molecules may be oxidized by mono- or dioxygenases into reactive compounds. An analogous biochemical process occurs in animals, catalyzed by cytochrome P450s [44,45], producing toxic and mutagenic electrophiles. ROS deriving from PAH oxidation would increase the overall ROS level, and thereby contribute towards activation of ROS-dependent signaling pathways. Alternatively, the PAH molecule may be directly recognized by a receptor such as a PAS-domain protein, a large and widely-distributed class of environmental sensors that includes the vertebrate aryl hydrocarbon receptor $[46,47]$. The strong similarities to biotic stress also suggest that the PAH could be cross-reacting with a pathogen recognition system. Regardless of the ini- tial mechanism of action, the hormones SA, ethylene, and JA appear to be involved in the response, and other unidentified signals also are likely relevant. Finally, the oxidized intermediates can be conjugated with a sugar or glutathione, and sequestered into the vacuole or cell wall. The initial PAH or its downstream products have been shown to accumulate in trichomes and other epidermal cells [14], although the recognition and transport mechanisms remain unknown.

Additional studies will help elucidate causality in the complex PAH stress response. It would be instructive to perform high-resolution time-series experiments to measure transcripts implicated in the earliest modes of action, as well as direct measurement of hormone levels. In addition, it would be valuable to perform tissue-specific molecular and enzyme assays, particularly of the zones implicated by the positive GUS results and necrotic areas. Furthermore, in addition to the ethylene mutants, the PAH response in other signaling mutants should be analysed.

Even though many remaining questions surround PAH stress, the microarray data provide a number of leads for improving $\mathrm{PAH}$ phytoremediation. Relaxing the rate- and capacity-limiting bottlenecks in the PAH detoxification pathway would reduce the cytoplasmic concentration of PAHs, thereby decreasing the effective toxicity to the plant and allowing increased uptake of the pollutant. For example, further increasing GST and UGT protein levels, or artificially up-regulating vacuolar transporters of conjugated xenobiotics, may produce plants with improved phytoremediation capabilities. The present results, as well as the suggested follow-on research, will be of great value in breeding and engineering plants for phytoremediation of polycyclic aromatic hydrocarbons.

\section{Conclusions}

The microarray experiments and comparative analyses show that phenanthrene treatment of Arabidopsis induces oxidative stress networks, closely resembling pathogen defense programs. A battery of altered transcripts revealed perturbations of the ROS, HR, and SAR systems. The present data support the hypothesis that the hormones SA, ethylene, and JA are involved in PAH response. In total, the results provide a large number of new pathway targets for researching and engineering plants for PAH phytoremediation.

\section{Methods}

\section{Plants and Growth Conditions}

Seeds of the Arabidopsis ecotype Colombia were obtained from Arabidopsis Research Centre and used as the WT control in all experiments. Seeds of the mutants eto3, etr1-7, etr1-4, and etr1-6;etr2-3;ein4-4 were a gift from Eric Schaller or were obtained from ABRC. Seeds of 
AtGSTF2::GUS fusion plants [19] were a gift from Peter Goldsbrough. Seeds of bean basic chitinase CH5B::GUS fusion plants [31] were a gift from Sara Patterson.

Seeds were surface-sterilized, stratified, and placed in Petri dishes containing half-strength Murashige and Skoog medium, supplemented with sucrose and $0,0.25$ or $0.5 \mathrm{mM}$ of phenanthrene, as described previously [14]. ACC was added to the growth medium in appropriate amounts before autoclaving. Plants were grown at $23 \pm$ $1^{\circ} \mathrm{C}$ either in the dark or under long-day conditions $(16 / 8$ h photoperiod at approximately $130 \mu \mathrm{mol}$ photons $\left.m^{-2} s^{-1}\right)$ for 4-21 d as indicated in the text. Before plates were put in darkness, they were exposed to white light for 10-12 h to achieve uniform germination. When root or hypocotyl lengths were to be measured, plates were kept in vertical orientation. Each plate contained seeds of the WT Columbia and at least of one mutant. Plants were observed under a Zeiss 2000-C dissection microscope equipped with an Olympus 340 digital camera.

All experiments were conducted at least twice with each mutant, with at least ten plants of each genotype per treatment.

\section{DNA Microarray Analysis}

PAH treated ( $0.25 \mathrm{mM}$ phenanthrene) and control plants (0 $\mathrm{mM}$ phenanthrene) were grown under long days and harvested at $21 \mathrm{~d}$, and at least 20 plants were pooled and stored at $-80^{\circ} \mathrm{C} .500 \mathrm{mg}$ tissue was removed from each pool and RNA was isolated using TRIzol (Molecular Research Center) per the manufacturer's instructions. Resulting samples were treated with DNase I (Invitrogen) and purified with RNeasy Mini Cleanup (Qiagen) per the manufacturers' instructions. Labeling was performed with the Affymetrix Enzo kit and processed on a Affymetrix Fluidics Station Model 450. Hybridized chips were read on a model M10 scanner.

Two rounds of biological replication were analyzed. In the first replicate, treated and control samples were each run on one Affymetrix ATH1-121501 microarray. In the second biological replicate, the treated sample was applied to one microarray, and the control sample was applied to two microarrays as a technical replicate. See Additional File 7, Additional File 8, and Additional File 9 for further technical details on the microarray experiment.

Validating the microarray data, previous RT-PCR analysis of actin-7, eif4a, PR-1, PDF1.2b, and AtEXP8 [14] (and unpublished data) are consistent with the present results. In addition, using RT-qPCR with four replicates per reaction and actin- 7 as a reference, we validated that the differential responses for GSTF6 and PR-1 are consistent with the microarray dataset (data not shown).

\section{Bioinformatic Analyses}

Data analysis was performed in R version 2.9.2 [48] and Bioconductor version 2.4.1 [49] installed on x86 hardware running Debian Linux Version 5.0. All of the procedures below were scripted in $\mathrm{R}$ and Python software written for this project.

To determine differential expression of the phenanthrene microarray dataset, the Affymetrix .CEL files were normalized by the Bioconductor just.gcrma algorithm using default parameters [50]. To reduce the false discovery rate, nonspecific prefiltering was performed using the Bioconductor genefilter package, eliminating probes with raw signal intensity less than 100 on all microarrays, and eliminating probes with an interquartile intensity ratio of less than 1.41 across the microarrays. The prefiltered set was then tested for statistical significance by a linear model using Limma [51], corrected for multiple comparisons with a Benjamini and Hochberg false discovery rate limit of 0.05 . To identify genes with putative biological significance, probes with differential expression ratios greater than 2-fold up or 2-fold down were preserved, and these remaining probes were defined as the set of 1031 differentially-expressed, phenanthrene responsive genes used in subsequent analysis. The Affymetrix probe identifiers were mapped to Arabidopsis Genome Identifiers (AGIs), symbols, and annotations using the ath1121501.db metadata in Bioconductor.

To compare the phenanthrene microarray data with published microarray data, Affymetrix ATH1 .CEL files were obtained from the AffyWatch service of the Nottingham Arabidopsis Stock Centre http://affymetrix.arabidopsis.info. The published .CEL files and our phenanthrene .CEL files were normalized together using just . gcrma as described above. To perform the hierarchical clustering shown by the heatmap, Kendall tau correlation matrices between genes and experiments were computed, and complete linkage clustering was computed by the $\mathrm{R}$ hclust function. The resulting clustering was visualized by the $\mathrm{R}$ heatmap. 2 algorithm.

Gene ontology analysis for overrepresented biological process (BP) terms was performed with the GOstats package of Bioconductor [52]. The set of 1031 differentially-expressed probes was partitioned into up-regulated and down-regulated subsets, and their Affymetrix probe identifiers were mapped to Arabidopsis Genome Identifiers (AGI). These AGI sets were tested against the universe of probed AGIs using the hyperGTest function, using a p-value cutoff of 0.05 and with the conditional scoring algorithm enabled.

MapMan [53] maps were produced to visualize cellular processes affected by the phenanthrene treatment. $\log _{2}-$ transformed mean differences between transcript signals 
in phenanthrene-treated and control microarrays served as input to MapMan.

\section{Root and Shoot Measurements}

Root and shoot lengths were measured on digital photographs using NIH ImageJ v 1.3.1_13 software [54]. In each experiment phenanthrene response percentage (R) of a genotype was calculated as $R=100 \times\left(A V E_{p} / A V E_{c}\right)$, where $A V E_{p}$ is the mean organ length in phenanthrene treatment and $A V E_{c}$ is the mean organ length in control (i.e., in absence of phenanthrene). Error of R (RE) was calculated as $R E=R \times \sqrt{\left(\frac{\delta_{c} / \sqrt{n_{c}}}{A V E_{c}}\right)^{2}+\left(\frac{\delta_{p} / \sqrt{n_{p}}}{A V E_{p}}\right)^{2}}$, where $\delta_{c}$ and $\delta_{p}$ are standard deviations of organ length in control and phenanthrene treatment, respectively; $n_{c}$ and $n_{p}$ are numbers of roots or hypocotyls measured in control and phenanthrene treatment, respectively. In the root and hypocotyl length assay mutant's response to phenanthrene $R_{m}$ was considered significantly different from the WT response (R $[\mathrm{wt}])$, if the intervals $\left[R_{w t}-R E_{w t}, R_{w t}+\right.$ $\left.R E_{w t}\right]$ for the WT, and $\left[R_{m}-R E_{m}, R_{m}+R E_{m}\right]$ for the mutant, did not overlap.

\section{Histochemistry}

GUS-staining was performed as described by [55]. To facilitate relative comparisons of reporter activity, identical GUS staining conditions were used in all experiments. For all histochemical methods whole plants or shoots were photographed under a Zeiss 2000-C dissecting microscope equipped with an Olympus 340 digital camera before and after staining. Images representative of at least ten plants per treatment and experiment are shown in Figure 3 and Figure 4.

\section{Additional material}

Additional file 1 Full microarray dataset. This .csv file contains the full, non-prefiltered microarray experiment dataset. All values were $\log _{2}$ transformed before calculations. Ratio, replicate means, and p-values were computed by the Bioconductor limma package.

Additional file 2 Differentially-expressed transcripts. This .csv file contains the set of 1031 differentially-expressed transcripts, computed as described in the Methods section. Ratio values are $\log _{2}$ transformed.

Additional file $\mathbf{3}$ Gene Ontology Summary. This .html file describes the results of the gene ontology analysis, performed separately on the sets of differentially-expressed upregulated and downregulated transcripts. The diffExpr column represents the $\log _{2}$ transformed values.
Additional file 4 MapMan illustrations of phenanthrene-responsive cellular processes and pathways. Phenanthrene responsive cellular processes and pathways in Arabidopsis. MapMan illustrations of transcripts in plants grown on $0.25 \mathrm{mM}$ phenanthrene for $21 \mathrm{~d}$ as compared to transcript levels in untreated control plants. Figure a: Overview of metabolism; Figure b: Overview of photosynthesis; Figure c: Overview of carotenoid biosynthesis; Figure d: Overview of protein targeting; Figure e: Overview of cellular responses; Figure f: Overview of gene regulation. Signal colors: Red downregulated, blue, upregulated transcripts in phenanthrene-treated plants. Scale values represent the differences between the mean $\log _{2}$-transformed values of the treated and untreated microarray sets.

Additional file $\mathbf{5}$ Phenanthrene induced changes in gene expression. Arabidopsis seedlings were grown in absence (CTR) or presence (PHE) of $0.25 \mathrm{mM}$ phenanthrene for 21 days and total RNA was extracted. Microarray analysis was carried out as described in the Methods section. Columns CTR (mean microarray signal from control plants), PHE (mean microarray signal from phenanthrene-treated plants), and Fold-change (PHE/CTR) are $\log _{2}$ transformed.

Additional file 6 Heatmap gene details. This .html file details the contents of Figure 2. Prior to clustering, the full set of microarrays was batchnormalized as described in the Methods section; consequently, the phenanthrene experiment microarray values in this file differ slightly from the values elsewhere in this report.

Additional file $\mathbf{7}$ Microarray quality control analysis. This file contains a quality control analysis of the raw microarray data used in this study. The analysis was produced using the Bioconductor package arrayQualityMetrics. Jun04 no phe.cel Jun04 phe.cel represent the untreated control and phenanthrene-treated samples, respectively, of the first replicate experiment. From the second replicate experiment, Aug04_no_phe_A.cel and Aug04_no_phe_C.cel represent the control, and Aug04_phe_B.cel represents the treated sample.

Additional file $\mathbf{8}$ Microarray volcano plot. The volcano plot represents the dataset from the five microarray chips after gcRMA normalization and linear model processing by the Bioconductor limma package.

Additional file $\mathbf{9}$ Minimum information about a microarray experiment (MIAME) checklist. The minimum information about a microarray experiment (MIAME) data is supplied in Additional File 9.

\section{Authors' contributions}

DW performed the microarray experiments and bioinformatics analyses. MA performed the mutant growth and histochemistry experiments. DW and MA contributed equally to this work. ACC conceived of the studies and contributed to the planning and oversight of the experiments. All authors contributed to the data analysis and composition of the manuscript.

\section{Acknowledgements}

This work was supported by the University of Massachusetts Boston, and by a Joint Interagency Program on Phytoremediation Research grant from the National Science Foundation (grant no. IBN-0343856) to ACC. We thank Roderick Jensen for helpful discussions and his laboratory at University of Massachusetts Boston for carrying out the labeling, hybridization and scanning procedures of the microarray experiment.

\section{Author Details}

1Department of Biology, University of Massachusetts Boston, 100 Morrissey Blvd, Boston, MA 02125, USA and 2Institute of Biological Production Systems, Fruit Science Section, Leibniz University Hannover, Herrenhäuser Str 2, D-30419 Hannover, Germany

Received: 2 October 2009 Accepted: 7 April 2010 Published: 7 April 2010

\section{References}

1. Meudec A, Poupart N, Dussauze J, Deslandes E: Relationship between heavy fuel oil phytotoxicity and polycyclic aromatic hydrocarbon contamination in Salicornia fragilis. Sci Total Environ 2007, 381:146-156.

2. Oros DR, Ross JRM, Spies RB, Mumley T: Polycyclic aromatic hydrocarbon (PAH) contamination in San Francisco Bay: a 10-year retrospective of monitoring in an urbanized estuary. Environ Res 2007, 105:101-118. 
3. Luch A: Nature and nurture - Lessons from chemical carcinogenesis. Nat Rev Cancer 2005, 5:113-125.

4. US Dept of Health and Human Services, Public Health Service, National Toxicology Program: Report on carcinogens. 2005 [http:// ntp.niehs.nih.gov/ntp/roc/eleventh/profiles/s150pah.pdf]

5. Pilon-Smits E: Phytoremediation. Annu Rev Plant Biol 2005, 56:15-39.

6. Harvey PJ, Campanella BF, Castro PML, Harms H, Lichtfouse E, Schäffner AR, Smrcek S, Werck-Reichhart D: Phytoremediation of polyaromatic hydrocarbons, anilines and phenols. Environ Sci Pollut Res Int 2002، 9:29-47.

7. Dhankher OP, Li Y, Rosen BP, Shi J, Salt D, Senecoff JF, Sashti NA, Meagher RB: Engineering tolerance and hyperaccumulation of arsenic in plants by combining arsenate reductase and gamma-glutamylcysteine synthetase expression. Nat Biotechnol 2002, 20:1140-1145.

8. Li Y, Dankher OP, Carreira L, Smith AP, Meagher RB: The shoot-specific expression of gamma-glutamylcysteine synthetase directs the longdistance transport of thiol-peptides to roots conferring tolerance to mercury and arsenic. Plant Physiol 2006, 141:288-298.

9. Dhankher OP, Rosen BP, McKinney EC, Meagher RB: Hyperaccumulation of arsenic in the shoots of Arabidopsis silenced for arsenate reductase (ACR2). Proc Natl Acad Sci USA 2006, 103:5413-5418.

10. Liu H, Weisman D, Ye Y, Cui B, Huang Y, Wang Z: An oxidative stress response to polycyclic aromatic hydrocarbon exposure is rapid and complex in Arabidopsis thaliana. Plant Sci 2009, 176:375-382.

11. Paskova V, Hilscherova K, Feldmannova M, Blaha L: Toxic effects and oxidative stress in higher plants exposed to polycyclic aromatic hydrocarbons and their N-heterocyclic derivatives. Environ Toxicol Chem 2006, 25:3238-3245

12. Burritt DJ: The polycyclic aromatic hydrocarbon phenanthrene causes oxidative stress and alters polyamine metabolism in the aquatic liverwort Riccia fluitans L. Plant Cell Environ 2008, 31:1416-1431.

13. Gao Y, Zhu L: Plant uptake, accumulation and translocation of phenanthrene and pyrene in soils. Chemosphere 2004, 55:1 169-1178.

14. Alkio M, Tabuchi TM, Wang XC, Colon-Carmona A: Stress responses to polycyclic aromatic hydrocarbons in Arabidopsis include growth inhibition and hypersensitive response-like symptoms. J Exp Bot 2005, 56:2983-2994.

15. Langebartels C, Wohlgemuth H, Kschieschan S, Grün S, Sandermann H: Oxidative burst and cell death in ozone-exposed plants. Plant Physiol Biochem 2002, 40:567-575.

16. Pierik R, Tholen D, Poorter H, Visser EJW, Voesenek LACJ: The Janus face of ethylene: growth inhibition and stimulation. Trends Plant Sci 2006, 11:176-183.

17. Broekaert WF, Delauré SL, Bolle MFCD, Cammue BPA: The role of ethylene in host-pathogen interactions. Annu Rev Phytopathol 2006, 44:393-416.

18. Lin Z, Zhong S, Grierson D: Recent advances in ethylene research. J Exp Bot 2009, 60:3311-3336.

19. Smith AP, Nourizadeh SD, Peer WA, Xu JH, Bandyopadhyay A, Murphy AS, Goldsbrough PB: Arabidopsis AtGSTF2 is regulated by ethylene and auxin, and encodes a glutathione S-transferase that interacts with flavonoids. Plant J 2003, 36:433-442.

20. Potter S, Uknes S, Lawton K, Winter AM, Chandler D, Dimaio J, Novitzky R, Ward E, Ryals J: Regulation of a hevein-like gene in Arabidopsis. Mol Plant Microbe Interact 1993, 6:680-685.

21. Penninckx I, Eggermont K, Terras FRG, Thomma B, DeSamblanx GW, Buchala A, Metraux JP, Manners JM, Broekaert WF: Pathogen-induced systemic activation of a plant defensin gene in Arabidopsis follows a salicylic acid-independent pathway. Plant Cell 1996, 8:2309-2323.

22. Marrs KA: The functions and regulation of glutathione S-transferases in plants. Annu Rev Plant Physiol Plant Mol Biol 1996, 47:127-158.

23. Wagner U, Edwards R, Dixon DP, Mauch F: Probing the diversity of the Arabidopsis glutathione S-transferase gene family. Plant Mol Biol 2002, 49:515-532.

24. Mezzari MP, Walters K, Jelínkova M, Shih MC, Just CL, Schnoor JL: Gene expression and microscopic analysis of Arabidopsis exposed to chloroacetanilide herbicides and explosive compounds. A phytoremediation approach. Plant Physiol 2005, 138:858-869.

25. Gadjev I, Vanderauwera S, Gechev TS, Laloi C, Minkov IN, Shulaev V, Apel K, Inzé D, Mittler R, Breusegem FV: Transcriptomic footprints disclose specificity of reactive oxygen species signaling in Arabidopsis. Plant Physiol 2006, 141:436-445.
26. lida K, Seki M, Sakurai T, Satou M, Akiyama K, Toyoda T, Konagaya A, Shinozaki K: RARTF: Database and tools for complete sets of Arabidopsis transcription factors. DNA Res 2005, 12:247-256.

27. Lorenzo O, Piqueras R, Sanchez-Serrano JJ, Solano R: Ethylene response factor 1 integrates signals from ethylene and jasmonate pathways in plant defense. Plant Cell 2003, 15:165-178

28. Miller G, Shulaev V, Mittler R: Reactive oxygen signaling and abiotic stress. Physiol Plant 2008, 133:481-489.

29. Durrant WE, Dong X: Systemic acquired resistance. Annu Rev Phytopathol 2004, 42:185-209.

30. Rodríquez Fl, Esch JJ, Hall AE, Binder BM, Schaller GE, Bleecker AB: A copper cofactor for the ethylene receptor ETR1 from Arabidopsis. Science 1999, 283:996-998.

31. Chen QHG, Bleecker AB: Analysis of ethylene signal-transduction kinetics associated with seedling-growth response and chitinase induction in wild-type and mutant Arabidopsis. Plant Physiol 1995, 108:597-607.

32. Woeste KE, Ye C, Kieber JJ: Two Arabidopsis mutants that overproduce ethylene are affected in the posttranscriptional regulation of 1 aminocyclopropane-1-carboxylic acid synthase. Plant Physiol 1999, 119:521-530.

33. Hall AE, Grace Chen Q, Findell JL, Eric Schaller G, Bleecker AB: The relationship between ethylene binding and dominant insensitivity conferred by mutant forms of the etr 1 ethylene receptor. Plant Physiol 1999, 121:291-300.

34. Hua J, Meyerowitz EM: Ethylene responses are negatively regulated by a receptor gene family in Arabidopsis thaliana. Cell 1998, 94:261-271.

35. Cancel JD, Larsen PB: Loss-of-function mutations in the ethylene receptor ETR1 cause enhanced sensitivity and exaggerated response to ethylene in Arabidopsis. Plant Physiol 2002, 129:1557-1567.

36. Tosti N, Pasqualini S, Borgogni A, Ederli L, Falistocco E, Crispi S, Paolocci F: Gene expression profiles of O3-treated Arabidopsis plants. Plant Cell Environ 2006, 29:1686-1702.

37. Wohlgemuth $H$, Mittelstrass K, Kschieschan S, Bender J, Weigel H, Overmyer K, Kangasjärvi J, Sandermann H, Langebartels C: Activation of an oxidative burst is a general feature of sensitive plants exposed to the air pollutant ozone. Plant Cell Environ 2002, 25:717-726.

38. Xiong L, Zhu JK: Molecular and genetic aspects of plant responses to osmotic stress. Plant Cell Environ 2002, 25:131-139.

39. Mahalingam R, Fedoroff $\mathrm{N}$ : Stress response, cell death and signalling: the many faces of reactive oxygen species. Physiol Plant 2003, 119:56-68.

40. Kotchoni SO, Gachomo EW: The reactive oxygen species network pathways:an essential prerequisite for perception of pathogen attack and the acquired disease resistance in plants. J Biosci 2006, 31:389-404.

41. Overmyer K, Brosché M, Kangasjärvi J: Reactive oxygen species and hormonal control of cell death. Trends Plant Sci 2003, 8:335-342.

42. Ludwikow A, Gallois P, Sadowski J: Ozone-induced oxidative stress response in Arabidopsis: transcription profiling by microarray approach. Cell Mol Biol Lett 2004, 9:829-842.

43. Lieberherr D, Wagner U, Dubuis PH, Métraux JP, Mauch F: The rapid induction of glutathione S-transferases AtGSTF2 and AtGSTF6 by avirulent Pseudomonas syringae is the result of combined salicylic acid and ethylene signaling. Plant Cell Physiol 2003, 44:750-757.

44. Nota B, Bosse M, Ylstra B, van Straalen NM, Roelofs D: Transcriptomics reveals extensive inducible biotransformation in the soil-dwelling invertebrate Folsomia candida exposed to phenanthrene. $B M C$ Genomics 2009, 10:236.

45. Baird WM, Hooven LA, Mahadevan B: Carcinogenic polycyclic aromatic hydrocarbon-DNA adducts and mechanism of action. Environ $\mathrm{Mol}$ Mutagen 2005, 45:106-114.

46. Hefti MH, Francoijs KJ, de Vries SC, Dixon R, Vervoort J: The PAS fold. A redefinition of the PAS domain based upon structural prediction. Eur J Biochem 2004, 271:1198-1208.

47. Gu YZ, Hogenesch JB, Bradfield CA: The PAS superfamily: sensors of environmental and developmental signals. Annu Rev Pharmacol Toxicol 2000, 40:519-561.

48. R Development Core Team: R: A Language and Environment for Statistical Computing 2009 [http://www.R-project.org]. R Foundation for Statistical Computing, Vienna, Austria [ISBN 3-900051-07-0].

49. Gentleman RC, Carey VJ, Bates DM, Bolstad B, Dettling M, Dudoit S, Ellis B, Gautier L, Ge Y, Gentry J, Hornik K, Hothorn T, Huber W, lacus S, Irizarry R, 
Leisch F, Li C, Maechler M, Rossini AJ, Sawitzki G, Smith C, Smyth G, Tierney $L$, Yang JY, Zhang J: Bioconductor: open software development for computational biology and bioinformatics. Genome Biol 2004, 5:R80.

50. Wu Z, Irizarry RA, Gentleman R, Martinez-Murillo F, Spencer F: A modelbased background adjustment for oligonucleotide expression arrays. $J$ Am Stat Assoc 2004, 99:909-917.

51. Smyth GK: Linear models and empirical bayes methods for assessing differential expression in microarray experiments. Stat Appl Genet Mol Biol 2004, 3:Article3.

52. Falcon S, Gentleman R: Using GOstats to test gene lists for GO term association. Bioinformatics 2007, 23:257-258

53. Thimm O, Bläsing O, Gibon Y, Nagel A, Meyer S, Krüger P, Selbig J, Müller $L A$, Rhee SY, Stitt M: MAPMAN: a user-driven tool to display genomics data sets onto diagrams of metabolic pathways and other biological processes. Plant J 2004, 37:914-939.

54. Rasband W: ImageJ: Image Processing and Analysis in Java. 2004 [http:/ /rsb.info.nih.gov/ij/ National Institutes of Health, Bethesda, Maryland, USA

55. Jefferson RA, Kavanagh TA, Bevan MW: Gus fusions - beta-glucuronidase as a sensitive and versatile gene fusion marker in higher-plants. Embo Journal 1987, 6:3901-3907.

doi: 10.1186/1471-2229-10-59

Cite this article as: Weisman et al., Transcriptional responses to polycyclic aromatic hydrocarbon-induced stress in Arabidopsis thaliana reveal the involvement of hormone and defense signaling pathways BMC Plant Biology 2010, 10:59

Submit your next manuscript to BioMed Central and take full advantage of:

- Convenient online submission

- Thorough peer review

- No space constraints or color figure charges

- Immediate publication on acceptance

- Inclusion in PubMed, CAS, Scopus and Google Scholar

- Research which is freely available for redistribution

Submit your manuscript at www.biomedcentral.com/submit
Ciomed Central 